\title{
Effect of sunnhemp (Crotalaria juncea L.) green manuring on weed dynamics in puddled transplanted rice-based systems
}

\author{
Zabiullah Jalali ${ }^{1}$ and Mohammad Hussain Falahzadah*2 \\ ${ }^{1}$ Agronomy Department, Agriculture Faculty, Kabul University Kabul, Afghanistan \\ 2 Plant Protection Department, Agriculture Faculty, Kabul University, Kabul, Afghanistan \\ Address: \\ *Corresponding Author: Mohammad H. Falahzadah, falahzadahm@gmail.com \\ Received: 21.07.2021; Accepted: 28.10.2021; Published: 30.10.2021
}

10.21608/ejar.2021.81709.1118

\section{ABSTRACT}

Effects of Crotalaria juncea green manure on weed dynamics in transplanted rice-based systems were investigated in a field study conducted over two cropping seasons, 2016 and 2017, at Ludhiana, India. The major weed species in the field, during the green manuring phase, included Cyperus rotundus, Digitaria ciliaris and Eleusine indica. The use of cover/green manure crops and their residues to suppress weeds has shown potential to manage weeds. In this context, green manuring/cover cropping has great potential and is feasible in a rice-wheat system in northern India as there is a 45 to 60 days' fallow period between wheat harvest and transplanting of rice. C. juncea with 50 and $100 \mathrm{~kg}$ seed ha-1 recorded significantly lower density and biomass of aerobics weeds and weed density as compared to without green manure in both years; GM with $100 \mathrm{~kg}$ seed ha-1 had a more suppressing effect on weeds than green manuring with $50 \mathrm{~kg}$ seed ha-1. The residual effects of weed control treatments, applied during 2016 to succeeding transplanted rice crops, did not have any specific trend on weed density in 2017. The major weeds in green manuring crop were $C$. rotundus, D. ciliaris and $E$. indica. But in transplanted rice, Echinochloa colona, E. crus-galli and Leptochloa chinensis were major weeds.

Keywords: Green manure, weed dynamics, aerobic weeds, rice

\section{INTRODUCTION}

Green manures in annual crop rotations can bring significant benefits to global cropping systems. Biological $\mathrm{N}$ fixation has a lot of benefits to plant legume green manures or cover crops (Martens and Entz, 2011; Chen et al., 2018; Setyorini and Saraswati, 2019; Gao et al., 2021). Nitrogen management is a major concern for farmers because $\mathrm{N}$ is the nutrient required by the majority of crops in maximum amounts and is also easily removed from the system. Historically, biological $\mathrm{N}$ fixation has been supplied by rhizobium and other bacteria in communication with most legumes $\mathrm{N}$ needed in cropping systems (Raviv, 2015; Rathore et al., 2020). Its use has been widespread since Haber-Bush invented synthetic nitrogen fertilizers in the early 20th century (Kumar et al., 2018; (He et al., 2020; Gao, et al., 2020). The green manure and cover crops improve soil structure and organic matter (Gao et al., 2020; Marshall and Lynch, 2020; Sheng-nan et al., 2018), suppress weeds and reduces weed seed bank (Kumar and Ladha, 2011; Pratt et al., 2016; Songjuan et al., 2021). The use of smother crops or cover crop residue to suppress weed growth in agriculture is not a recent innovation; yet, only recently have smother, or cover crops received considerable attention. The cover crops offer environmental advantages that promote farm sustainability.

Even if there is a direct $\mathrm{N}$ advantage for the following crop, growing annual legume green manure crops is generally not seen as an economically viable option by ordinary farmers (Recalde et al., 2015; Longa et al., 2017; Martens et al., 2019). Lack of direct income from green manure also faces economic challenges for organic producers. In this situation, green bean manure can increase the adoption of this practice in both organic and conventional production systems (Chauhan and Johnson, 2011; Ghosh et al., 2007). Green manuring has been practised before rice transplanting, in northern India. Apart from its benefit to soil health, green manure also suppresses weeds and reduces weed seed bank (Kumar and Ladha, 2011; Qaswar et al., 2019). Green manuring is more effective on weed species present in top soil layers, light responsive and having low dormancy (Chauhan and Johnson, 2008). Sunnhemp (Crotalaria juncea L.) has been known for larger weed suppression than other crops, its plants make vigorous growth and shade the weed plants (Raheem et al., 2019; Deepanshi et al., 2020).

The weed floras of puddle transplanted rice consist of annual grasses, sedges and broad-leaf weeds. In rice, weeds could reduce the grain yield by 33-45\% (Singh et al., 2007; Manhas et al., 2012; Maguire et al., 2020). The herbicides have been major tool used for weed control in rice in northern India, and herbicide use is likely to increase further with rising labour costs and labour scarcity. Higher dependence on herbicides results in weed flora shift and herbicide resistance (Couëdel et al., 2018; Müjdecı et al., 2020; Zhou et al., 2020). Hence, non-chemical practices which can reduce the early weed pressure in soil and provide a favorable environment for crops need to be identified and incorporated in weed management programs. The present study investigated the effect of sunnhemp green manuring on weed dynamics in transplanted rice-based systems.

\section{MATERIALS AND METHODS:}

The field experiment was conducted in the summer seasons of 2016 and 2017 at the research farm of Punjab Agricultural University, Ludhiana, India which is situated in Trans-Gangetic Agro-Climatic zone, representing the Indo-Gangetic Alluvial 
Plain at $30^{\circ} 56^{\prime} \mathrm{N}$ latitude, $75^{\circ} 52^{\prime} \mathrm{E}$ longitude and an altitude of $247 \mathrm{~m}$ above mean sea level.

Soil characteristics:

The experimental soil was loamy sand, pH (7.1), EC (0.20 dS m-1), low in organic carbon (0.31\%), extractable N (251 kg ha-1), extractable $\mathrm{P}\left(14 \mathrm{~kg} \mathrm{ha}^{-1}\right)$ and extractable $\mathrm{K}\left(165 \mathrm{~kg} \mathrm{ha}^{-1}\right)$.

Experimental details:

After the harvest of wheat, the seedbed was prepared and the layout of the trial was done. The green manure (Sunnhemp) crop was raised as per recommended practices of Punjab Agricultural University and after its incorporation at 6-8 weeks after sowing; rice crop was raised after transplanting the seedlings. After harvest of the preceding wheat crop, the field was irrigated. When the field came to optimum soil moisture, it was ploughed with one disc harrow and one cultivator. The seed of $C$. juncea was broadcast uniformly in the green manure treatment plots. The green manure crop was incorporated at 45 days after sowing with tractor drew disc harrow. After the incorporation of green manure, all the experimental plots were irrigated and puddling (wet tillage) was done with tractor operated puddler for transplanting of rice seedlings. Thirty days old rice seedlings (cv. PR 121; $125 \mathrm{~d}$ ) were transplanted, in a puddle field, in rows spaced at $20 \mathrm{~cm}$ and rice seedlings spaced at $15 \mathrm{~cm}$ on 25 June 2016 and 28 June 2017. Nitrogen at $187.5 \mathrm{~kg} \mathrm{ha}^{-1}$ was applied through Urea, in three equal splits, at 15, 25 and 40 DAYS. The field also received $62.5 \mathrm{~kg} \mathrm{ZnSO}_{4}$ ha-1 $^{-1}$ (Zinc sulphate heptahydrate 21 per cent) at the time of field preparation as basal dose. Weed control treatments were applied in the plots as per the treatments. Penoxsulam $22.5 \mathrm{~g} \mathrm{ha}^{-1}$ was sprayed 12 days after transplanting as per the treatments. The herbicide was sprayed with a knapsack sprayer fitted with flat fan nozzle using 375 litres of water ha-1. The water was drained out from the field before spray. In weed-free treatments, the plots were kept free from weeds by hand weeding as and when needed. All the plots were kept ponded about $10 \mathrm{~cm}$ deep water for the first two weeks of transplanting for proper establishment of rice seedlings. Thereafter irrigations were applied two days after the ponded water has infiltrated into the soil. The irrigation was stopped 15 days before crop harvest. The crop was harvested manually, from 20 to 24 October during 2016 and 2017, when grains were manure and straw had turned yellow colour. The harvested crop was collected in the respective plots. Plant height was recorded from each five plot randomly selected plants. It was taken from the base of the plant to the base of the last fully opened leaves and it was recorded at 45, 90 days after transplanting until emergence of panicle. It was expressed as an average of five plants. Tillers were counted from middle rows from two spots of $50 \mathrm{~cm}$ row length in each plot 45 and 90 days after transplanting and of crop and expressed as some tillers $\mathrm{m}^{-2}$ Number of effective tillers (panicle bearing tillers) was counted at harvest from plants of $50 \mathrm{~cm} \times 50 \mathrm{~cm}$ area with the help of quadrat from each plot. The density was finally expressed as a number of effective tillers $\mathrm{m}^{-2}$.

\section{Panicle length:}

The five representative panicles were selected randomly from each plot and their length from neck node to the apex was measured and expressed in $\mathrm{cm}$. Finally, panicle length was expressed as an average of five panicles. Ten panicles were selected randomly from each plot to count the number of grains panicle ${ }^{-1}$. Ten panicles were selected randomly from each plot. The grains from those panicles were separated and average grain weight panicle ${ }^{-1}$ was recorded. After threshing the crop in each net plot, a representative sample of seeds was collected from bulk production of the entire plot.

\section{Thousand-grain weight:}

One thousand grains were count from the produce of each treatment, their weight (g) was recorded using electronic balance and expressed in (g). The sample was collected after the threshed produce sufficiently dried and brought to a moisture content of $14 \%$.

\section{Grain yield:}

Harvested crop produce from the net plot was threshed manually by beating them against the hard surface. The grain yield was recorded in $\mathrm{kg} \mathrm{plot}^{-1}$ then expressed to $\mathrm{t} \mathrm{ha}^{-1}$ at $14 \%$ grain moisture. Bundle weight was taken before threshing and strawweight were recorded after deducting grain weight from the whole bundle weight, which was then expressed in $t$ ha-1. Periodic weed count:

The species-wise weed count was recorded using a quadrate $(0.5 \mathrm{~m} \times 0.5 \mathrm{~m})$ at 45 and 90 days. The weed density was expressed in the number per square meter. The weeds present inside the randomly placed quadrate were cut at the above ground level at 45 and 90 days and dried in the hot air oven at $60^{\circ} \mathrm{C}$. The dry matter of weed is expressed in $\mathrm{g} \mathrm{m}^{-2}$.

\section{Weed seed bank studies:}

Weed seed bank study was done by taking a soil sample from each plot in $15 \mathrm{~cm}$ depth with the help of a core sampler of diameter $10.5 \mathrm{~cm}$ before sowing of green manuring, before transplanting rice, at rice harvest. The soil samples were washed using $0.2 \mathrm{~mm}$ sieve cloth to separate weed seeds and from the soil. The seed samples were transferred to Petri dishes lined with wet filter papers in a laboratory. Germination of weeds was recorded at weekly intervals until no germination occurred in Petri-dishes. Total weed germination was compiled for analysis, and the days were converted to viable seeds $\mathrm{m}^{-2}$.

\section{Statistical analysis:}

Two-way analysis of variance of split-plot GLM procedure was calculated using SAS (SAS Institute, 2002) software to evaluate differences between treatments; means were compared using Tukey's test for pair-wise comparison at $P=0.05$. LSD values were calculated to compare interaction means. Days effects on weed density and biomass were subjected to square root transformation before statistical analysis because of high variability. 


\section{RESULTS}

Weed density:

The major weed species in the field, during the green manuring phase, included Cyperus rotundus, Digitaria ciliaris and Eleusine indica in both years (Tables 1, 2).

Table 1: Effect of green manuring treatments on weed dynamics in 2016

\begin{tabular}{|c|c|c|c|c|c|c|c|c|c|c|c|c|}
\hline \multirow{3}{*}{$\begin{array}{l}\text { Green } \\
\text { manuring }\end{array}$} & \multicolumn{12}{|c|}{ Periodic weed density (No. $\mathrm{m}^{-2}$ ) } \\
\hline & \multicolumn{3}{|c|}{ Cyperus rotundus } & \multicolumn{3}{|c|}{ Digitaria ciliaris } & \multicolumn{3}{|c|}{ Eleusine indica } & \multicolumn{3}{|c|}{ Total } \\
\hline & $\begin{array}{c}15 \\
\text { Days }\end{array}$ & $\begin{array}{c}30 \\
\text { Days }\end{array}$ & $\begin{array}{c}45 \\
\text { Days }\end{array}$ & $\begin{array}{c}15 \\
\text { Days }\end{array}$ & $\begin{array}{c}30 \\
\text { Days }\end{array}$ & $\begin{array}{c}45 \\
\text { Days }\end{array}$ & $\begin{array}{c}15 \\
\text { Days }\end{array}$ & $\begin{array}{c}30 \\
\text { Days }\end{array}$ & $\begin{array}{c}45 \\
\text { Days }\end{array}$ & $\begin{array}{c}15 \\
\text { Days }\end{array}$ & $\begin{array}{c}30 \\
\text { Days }\end{array}$ & $\begin{array}{c}45 \\
\text { Days }\end{array}$ \\
\hline $\begin{array}{l}\text { Without } \\
\text { GM }\end{array}$ & $\begin{array}{l}5.51 \\
(31)\end{array}$ & $\begin{array}{l}9.82 \\
(99)\end{array}$ & $\begin{array}{l}9.58 \\
(93)\end{array}$ & $\begin{array}{l}3.64 \\
(13)\end{array}$ & $\begin{array}{l}4.10 \\
(17)\end{array}$ & $\begin{array}{l}4.19 \\
(18)\end{array}$ & $\begin{array}{c}3.05 \\
(9)\end{array}$ & $\begin{array}{l}4.45 \\
(20)\end{array}$ & $\begin{array}{c}2.69 \\
(8)\end{array}$ & $\begin{array}{l}7.64 \\
(59)\end{array}$ & $\begin{array}{l}12.11 \\
(149)\end{array}$ & $\begin{array}{l}11.44 \\
(132)\end{array}$ \\
\hline $\begin{array}{l}\text { GM with } 50 \\
\mathrm{~kg} \quad C \text {. } \\
\text { juncea } \mathrm{ha}^{-1}\end{array}$ & $\begin{array}{l}4.54 \\
(22)\end{array}$ & $\begin{array}{l}5.20 \\
(28)\end{array}$ & $\begin{array}{l}5.73 \\
(34)\end{array}$ & $\begin{array}{l}1.18 \\
(1)\end{array}$ & $\begin{array}{l}2.04 \\
(4)\end{array}$ & $\begin{array}{l}2.16 \\
(5)\end{array}$ & $\begin{array}{c}1.33 \\
(1)\end{array}$ & $\begin{array}{l}2.07 \\
(4)\end{array}$ & $\begin{array}{l}1.96 \\
(4)\end{array}$ & $\begin{array}{l}5.14 \\
(28)\end{array}$ & $\begin{array}{l}6.39 \\
(42)\end{array}$ & $\begin{array}{l}7.16 \\
(53)\end{array}$ \\
\hline $\begin{array}{l}\text { GM with } \\
100 \mathrm{~kg} \mathrm{C.} \\
\text { juncea } \mathrm{ha}^{-1}\end{array}$ & $\begin{array}{l}3.27 \\
(11)\end{array}$ & $\begin{array}{l}3.49 \\
(13)\end{array}$ & $\begin{array}{l}4.24 \\
(18)\end{array}$ & $\begin{array}{l}1.00 \\
(0)\end{array}$ & $\begin{array}{l}2.37 \\
(6)\end{array}$ & $\begin{array}{l}1.75 \\
(3)\end{array}$ & $\begin{array}{l}1.13 \\
(0)\end{array}$ & $\begin{array}{c}2.22 \\
(5)\end{array}$ & $\begin{array}{l}1.78 \\
(4)\end{array}$ & $\begin{array}{l}3.86 \\
(15)\end{array}$ & $\begin{array}{l}5.41 \\
(31)\end{array}$ & $\begin{array}{l}5.32 \\
(29)\end{array}$ \\
\hline $\begin{array}{l}\text { LSD } \\
(P=0.05)\end{array}$ & 0.21 & 0.36 & 0.63 & 0.05 & 0.57 & 0.55 & 0.14 & 0.11 & 0.63 & 0.52 & 1.21 & 0.71 \\
\hline
\end{tabular}

Parentheses are original values; days subjected to square root transformation before analysis

${ }^{*} \mathrm{Bl}=$ Before Incorporation $=45$ days after sowing

Table 2: Effect of green manuring and weed control treatments on weed dynamics in 2017

\begin{tabular}{|c|c|c|c|c|c|c|c|c|c|c|c|c|}
\hline \multirow{3}{*}{ Treatment } & \multicolumn{12}{|c|}{ Periodic weed density (No. $\mathrm{m}^{-2}$ ) } \\
\hline & \multicolumn{3}{|c|}{ Cyperus rotundus } & \multicolumn{3}{|c|}{ Digitaria ciliaris } & \multicolumn{3}{|c|}{ Eleusine indica } & \multicolumn{3}{|c|}{ Total } \\
\hline & $\begin{array}{c}15 \\
\text { Days }\end{array}$ & $\begin{array}{c}30 \\
\text { Days }\end{array}$ & $\begin{array}{c}45 \\
\text { Days }\end{array}$ & $\begin{array}{c}15 \\
\text { Days }\end{array}$ & $\begin{array}{c}30 \\
\text { Days }\end{array}$ & $\begin{array}{c}45 \\
\text { Days }\end{array}$ & $\begin{array}{c}15 \\
\text { Days }\end{array}$ & $\begin{array}{c}30 \\
\text { Days }\end{array}$ & $\begin{array}{c}45 \\
\text { Days }\end{array}$ & $\begin{array}{c}15 \\
\text { Days }\end{array}$ & $\begin{array}{c}30 \\
\text { Days }\end{array}$ & $\begin{array}{c}45 \\
\text { Days }\end{array}$ \\
\hline \multicolumn{13}{|l|}{ Green manuring } \\
\hline Without GM & $\begin{array}{l}5.42 \\
(30)^{*} \\
\end{array}$ & $\begin{array}{l}10.51 \\
(113) \\
\end{array}$ & $\begin{array}{l}10.21 \\
(105) \\
\end{array}$ & $\begin{array}{l}3.31 \\
(10) \\
\end{array}$ & $\begin{array}{l}4.26 \\
(18) \\
\end{array}$ & $\begin{array}{l}4.16 \\
(17) \\
\end{array}$ & $\begin{array}{c}2.91 \\
(8) \\
\end{array}$ & $\begin{array}{l}4.28 \\
(18) \\
\end{array}$ & $\begin{array}{c}2.73 \\
(8) \\
\end{array}$ & $\begin{array}{l}7.39 \\
(55) \\
\end{array}$ & $\begin{array}{l}12.56 \\
(160) \\
\end{array}$ & $\begin{array}{l}11.83 \\
(141) \\
\end{array}$ \\
\hline $\begin{array}{l}\text { GM with } 50 \mathrm{~kg} C \text {. } \\
\text { juncea } \mathrm{ha}^{-1}\end{array}$ & $\begin{array}{l}4.36 \\
(19) \\
\end{array}$ & $\begin{array}{l}5.95 \\
(37) \\
\end{array}$ & $\begin{array}{l}5.76 \\
(34)\end{array}$ & $\begin{array}{c}1.10 \\
(0)\end{array}$ & $\begin{array}{c}1.82 \\
(4)\end{array}$ & $\begin{array}{c}1.94 \\
(4)\end{array}$ & $\begin{array}{c}1.21 \\
(1) \\
\end{array}$ & $\begin{array}{c}1.83 \\
(4)\end{array}$ & $\begin{array}{c}1.82 \\
(4)\end{array}$ & $\begin{array}{l}4.61 \\
(22) \\
\end{array}$ & $\begin{array}{l}6.99 \\
(49) \\
\end{array}$ & $\begin{array}{l}7.05 \\
(51) \\
\end{array}$ \\
\hline $\begin{array}{l}\text { GM with } 100 \mathrm{~kg} C \text {. } \\
\text { juncea } \mathrm{ha}^{-1}\end{array}$ & $\begin{array}{l}3.15 \\
(11) \\
\end{array}$ & $\begin{array}{l}3.82 \\
(16)\end{array}$ & $\begin{array}{l}3.88 \\
(16) \\
\end{array}$ & $\begin{array}{c}1.00 \\
(0)\end{array}$ & $\begin{array}{c}2.16 \\
(5)\end{array}$ & $\begin{array}{c}1.87 \\
(4)\end{array}$ & $\begin{array}{c}1.00 \\
(0)\end{array}$ & $\begin{array}{c}2.21 \\
(5)\end{array}$ & $\begin{array}{c}1.61 \\
(3)\end{array}$ & $\begin{array}{l}3.44 \\
(12) \\
\end{array}$ & $\begin{array}{l}5.55 \\
(33) \\
\end{array}$ & $\begin{array}{l}5.13 \\
(28) \\
\end{array}$ \\
\hline $\operatorname{LSD}(P=0.05)$ & 0.30 & 0.48 & 0.63 & 0.11 & 0.59 & 0.41 & 0.13 & 0.31 & 0.29 & 0.23 & 0.28 & 0.62 \\
\hline \multicolumn{13}{|c|}{ Weed control } \\
\hline Weedy & $\begin{array}{l}4.34 \\
(19) \\
\end{array}$ & $\begin{array}{l}7.18 \\
(57) \\
\end{array}$ & $\begin{array}{l}6.76 \\
(53)\end{array}$ & $\begin{array}{c}1.59 \\
(2)\end{array}$ & $\begin{array}{c}2.47 \\
(7)\end{array}$ & $\begin{array}{c}2.32 \\
(7)\end{array}$ & $\begin{array}{c}1.79 \\
(3)\end{array}$ & $\begin{array}{c}2.47 \\
(7)\end{array}$ & $\begin{array}{c}1.86 \\
(4)\end{array}$ & $\begin{array}{l}4.93 \\
(26) \\
\end{array}$ & $\begin{array}{l}8.43 \\
(79)\end{array}$ & $\begin{array}{l}7.89 \\
(71)\end{array}$ \\
\hline Weed free & $\begin{array}{l}4.71 \\
(24) \\
\end{array}$ & $\begin{array}{l}6.11 \\
(43) \\
\end{array}$ & $\begin{array}{l}6.06 \\
(43) \\
\end{array}$ & $\begin{array}{c}2.01 \\
(6)\end{array}$ & $\begin{array}{c}2.49 \\
(8)\end{array}$ & $\begin{array}{c}2.46 \\
(8)\end{array}$ & $\begin{array}{c}1.49 \\
(2)\end{array}$ & $\begin{array}{c}2.49 \\
(8)\end{array}$ & $\begin{array}{c}2.23 \\
(6)\end{array}$ & $\begin{array}{l}5.47 \\
(35) \\
\end{array}$ & $\begin{array}{l}7.75 \\
(65)\end{array}$ & $\begin{array}{l}7.61 \\
(65) \\
\end{array}$ \\
\hline $\begin{array}{l}\text { Penoxsulam } 22.5 \\
\mathrm{~g} \mathrm{ha}^{-1}\end{array}$ & $\begin{array}{l}4.25 \\
(19) \\
\end{array}$ & $\begin{array}{l}7.27 \\
(57) \\
\end{array}$ & $\begin{array}{l}7.10 \\
(54) \\
\end{array}$ & $\begin{array}{c}1.75 \\
(3) \\
\end{array}$ & $\begin{array}{l}3.23 \\
(11) \\
\end{array}$ & $\begin{array}{l}3.03 \\
(10) \\
\end{array}$ & $\begin{array}{c}1.92 \\
(4) \\
\end{array}$ & $\begin{array}{l}3.25 \\
(11) \\
\end{array}$ & $\begin{array}{c}2.31 \\
(6) \\
\end{array}$ & $\begin{array}{l}5.11 \\
(28) \\
\end{array}$ & $\begin{array}{l}9.15 \\
(87) \\
\end{array}$ & $\begin{array}{l}8.57 \\
(77) \\
\end{array}$ \\
\hline $\begin{array}{l}\text { Penoxsulam } 22.5 \\
\mathrm{~g} \mathrm{ha}^{-1}+\mathrm{HW}\end{array}$ & $\begin{array}{l}3.95 \\
(18) \\
\end{array}$ & $\begin{array}{l}6.48 \\
(55) \\
\end{array}$ & $\begin{array}{l}6.56 \\
(56) \\
\end{array}$ & $\begin{array}{c}1.81 \\
(4) \\
\end{array}$ & $\begin{array}{l}2.80 \\
(10) \\
\end{array}$ & $\begin{array}{l}2.82 \\
(10) \\
\end{array}$ & $\begin{array}{c}1.62 \\
(2) \\
\end{array}$ & $\begin{array}{l}2.88 \\
(10) \\
\end{array}$ & $\begin{array}{c}1.82 \\
(4) \\
\end{array}$ & $\begin{array}{l}5.09 \\
(30)\end{array}$ & $\begin{array}{l}8.15 \\
(92)\end{array}$ & $\begin{array}{l}7.95 \\
(79)\end{array}$ \\
\hline $\operatorname{LSD}(\mathrm{P}=0.05)$ & 0.46 & 0.51 & 0.42 & 0.13 & 0.31 & 0.30 & 0.17 & 0.20 & 0.28 & 0.24 & 0.22 & 0.41 \\
\hline Interaction LSD & 0.79 & 0.89 & 0.73 & 0.22 & 0.53 & 0.52 & 0.29 & 0.34 & 0.49 & 0.41 & 0.38 & 0.71 \\
\hline
\end{tabular}

*Parentheses are original values; daysa subjected to square root transformation before analysis 
The highest density of all weeds species was recorded at 30 days after sunnhemp sowing. Green manuring (GM) significantly suppressed germination of all weed species as compared to without green manure, at all the stages of the record, both in 2016 and 2017. The seed rate of $100 \mathrm{~kg} \mathrm{ha}^{-1}$ had a more weed suppression effect than $50 \mathrm{~kg}$ seed ha-1. In the case of total weed density, GM treatments had significantly lower density than without green manuring; $100 \mathrm{~kg} \mathrm{seed} \mathrm{ha}^{-1}$ had significantly lower density than $50 \mathrm{~kg}$ seed ha-1. Total weed density and $C$. rotundus density were significantly lower under $100 \mathrm{~kg}$ seed ha1 at 15, 30 and 45 DAS than $50 \mathrm{~kg}$ seed ha $^{-1}$ and without green manuring in both years. In the case of Digitaria ciliaris and Eleusine indica, $100 \mathrm{~kg}$ seed ha-1 was at par with $50 \mathrm{~kg} \mathrm{seed} \mathrm{ha}^{-1}$ at all stages in both years. The residual effects of weed control treatments, applied during 2016, did not show any specific trend on weed density in 2017. The interaction effects revealed that at the time of incorporation of GM at 45 DAS in 2017, the GM with both seed rates had a significantly lower density of all three weed species under all weed control treatments (Tables 3, 4 and 5).

\section{Soil weed seed bank:}

Among green manure treatments, in 2016, GM-100 had a significantly higher seed bank of Echinochloa crus-galli at the time of incorporation (45 days; rice transplanting) while GM-50 had a significantly higher seed bank than without GM (Table 6). In case of E. colona, the seed bank results among GM treatments were non-significant, both at the time of rice transplanting and at rice harvest. Among weed control treatments, in 2016, penoxsulam plus HW had significantly higher seed bank of $E$. crus-galli than all other treatments at crop harvest; the differences among weed control treatments for seed bank of $E$. colona were non-significant.

Table 3: Effect of sunnhemp green manuring on periodic grass weed biomass in 2016.

\begin{tabular}{|l|c|c|c|}
\hline \multirow{2}{*}{ Green manuring } & \multicolumn{2}{|c|}{ Grass weed dry biomass (g m ${ }^{-2}$ ) } \\
\cline { 2 - 4 } & 15 Days & 30 Days & 45 Days \\
\hline Without GM & $2.98(8)^{*}$ & $12.81(165)$ & $12.05(146)$ \\
\hline GM with $50 \mathrm{~kg} \mathrm{C.} \mathrm{juncea} \mathrm{ha-1}$ & $1.61(2)$ & $5.94(38)$ & $4.44(21)$ \\
\hline GM with $100 \mathrm{~kg}$ C. juncea ha-1 & $1.71(2)$ & $5.39(32)$ & $3.89(17)$ \\
\hline LSD (P=0.05) & 0.24 & 0.65 & 0.29 \\
\hline
\end{tabular}

* daysa was square root transformed before analysis; parentheses are original means; GM- green manuring

Table 4: Effect of sunnhemp green manuring and weed control in rice on periodic grass weed biomass in 2017.

\begin{tabular}{|c|c|c|c|}
\hline \multirow[t]{2}{*}{ Treatment } & \multicolumn{3}{|c|}{ Grass weeds biomass $\left(\mathrm{g} \mathrm{m}^{-2}\right)$} \\
\hline & 15 Days & 30 Days & 45 Days \\
\hline \multicolumn{4}{|l|}{ Green manuring } \\
\hline Without GM & $2.97(8)^{*}$ & $12.94(168)$ & $12.07(147)$ \\
\hline GM with 50 kg $C$. juncea ha-1 & $1.53(1)$ & $5.81(38)$ & $4.78(24)$ \\
\hline GM with $100 \mathrm{~kg} C$. juncea $\mathrm{ha}^{-1}$ & $1.69(2)$ & $5.17(30)$ & $4.09(18)$ \\
\hline LSD $(P=0.05)$ & 0.23 & 1.07 & 0.49 \\
\hline \multicolumn{4}{|l|}{ Weed control } \\
\hline Weedy check & $2.22(4)$ & $7.57(76)$ & $7.05(70)$ \\
\hline Weed Free & $2.05(4)$ & $7.09(67)$ & $6.62(58)$ \\
\hline Penoxsulam $22.5 \mathrm{~g} \mathrm{ha}^{-1}$ & $2.25(4)$ & $9.57(95)$ & $7.81(70)$ \\
\hline Penoxsulam $22.5 \mathrm{~g} \mathrm{ha}^{-1}+\mathrm{HW}$ & $1.74(2)$ & $7.67(77)$ & $6.45(54)$ \\
\hline LSD $(P=0.05)$ & 0.15 & 0.43 & 0.31 \\
\hline Interaction LSD $(P=0.05)$ & 0.26 & 0.75 & 0.54 \\
\hline
\end{tabular}

* daysa was square root transformed before analysis; parentheses are original means; GM- green manuring 
Table 5: Effect of green manuring and weed control treatments on soil weed seed bank in 2016

\begin{tabular}{|c|c|c|c|c|}
\hline \multirow{3}{*}{ Treatments } & \multicolumn{4}{|c|}{ Weed seed bank (No. of seeds $\left.\mathrm{m}^{-2}\right)^{*}$} \\
\hline & \multicolumn{2}{|c|}{ Before rice transplanting } & \multicolumn{2}{|c|}{ At harvest } \\
\hline & E. crus-galli & E. colona & E. crus-galli & E. colona \\
\hline \multicolumn{5}{|l|}{ Green manuring } \\
\hline Without GM & 575 & 230 & 501 & 355 \\
\hline GM with $50 \mathrm{~kg} C$. juncea ha-1 $^{-1}$ & 460 & 230 & 620 & 384 \\
\hline GM with $100 \mathrm{~kg}$ C. juncea ha-1 & 690 & 230 & 483 & 423 \\
\hline LSD (P=0.05) & 40 & NS & 30 & NS \\
\hline \multicolumn{5}{|l|}{ Weed control } \\
\hline Weedy & - & - & 508 & 390 \\
\hline Weed free & - & - & 550 & 383 \\
\hline Penoxsulam $22.5 \mathrm{~g} \mathrm{ha}^{-1}$ & - & - & 380 & 372 \\
\hline Penoxsulam $22.5 \mathrm{~g} \mathrm{ha}^{-1}+\mathrm{HW}$ & - & - & 702 & 403 \\
\hline $\operatorname{LSD}(P=0.05)$ & - & - & 37 & NS \\
\hline Interaction LSD ( $\mathrm{P}=0.05)$ & - & - & 65 & 62 \\
\hline Initial weed seed bank & 805 & 620 & - & - \\
\hline
\end{tabular}

The lower density of weeds under GM treatments than without green manure may be attributed to the allelopathic and smothering effects of GM vegetation on weeds.

\section{Weed dry biomass:}

GM treatments accumulated significantly lower weed biomass than without green manure in both years at 15,30 and 45 days (Tables 6 and 8); at the time of incorporation, weed biomass under $100 \mathrm{~kg}$ seed ha-1 was significantly lower than under $50 \mathrm{~kg}$ seed ha ${ }^{-1}$ in both years.

Table 6: Effect of green manuring and weed control treatments on soil weed seed bank in 2017

\begin{tabular}{|c|c|c|c|c|c|c|}
\hline \multirow[t]{3}{*}{ Treatment } & \multicolumn{6}{|c|}{ Weed seed bank (No. of seeds $\mathrm{m}^{-2}$ ) } \\
\hline & \multicolumn{2}{|c|}{$\begin{array}{c}\text { Before sowing of green } \\
\text { manure }\end{array}$} & \multicolumn{2}{|c|}{ Before rice transplanting } & \multicolumn{2}{|c|}{ At rice harvest } \\
\hline & E. crus-galli & E. colona & E. crus-galli & E. colona & E. crusg-alli & E. colona \\
\hline \multicolumn{7}{|l|}{ Green manuring } \\
\hline Without GM & 1501 & 536 & 1276 & 429 & 654 & 589 \\
\hline GM with $50 \mathrm{~kg} C$. juncea ha $^{-1}$ & 1639 & 557 & 1393 & 446 & 692 & 622 \\
\hline GM with $100 \mathrm{~kg} C$. juncea ha-1 & 2593 & 432 & 2204 & 345 & 902 & 812 \\
\hline $\operatorname{LSD}(P=0.05)$ & 125 & 46 & 106 & 37 & 112 & 101 \\
\hline \multicolumn{7}{|l|}{ Weed control } \\
\hline Weedy & 2563 & 1043 & 2179 & 835 & 1754 & 1579 \\
\hline Weed free & 1667 & 463 & 1417 & 370 & 618 & 557 \\
\hline Penoxsulam $22.5 \mathrm{~g} \mathrm{ha}^{-1}$ & 1775 & 384 & 1509 & 307 & 463 & 417 \\
\hline Penoxsulam $22.5 \mathrm{~g} \mathrm{ha}^{-1}+\mathrm{HW}$ & 1639 & 143 & 1394 & 114 & 161 & 145 \\
\hline $\operatorname{LSD}(P=0.05)$ & 181 & 41 & 153 & 33 & 82 & 74 \\
\hline Interaction LSD ( $\mathrm{P}=0.05)$ & 313 & NS & 266 & NS & 142 & 128 \\
\hline
\end{tabular}


Table7: Effect of green manuring and weed control treatments on weed dry biomass in transplanted rice

\begin{tabular}{|c|c|c|c|c|c|c|c|c|}
\hline \multirow[t]{3}{*}{ Treatment } & \multicolumn{4}{|c|}{ Grass weed dry biomass $\left(\mathrm{g} \mathrm{m}^{-2}\right)$} & \multicolumn{4}{|c|}{ Sedge dry biomass ( $\mathrm{g} \mathrm{m}^{-2}$ ) } \\
\hline & \multicolumn{2}{|c|}{45 Days } & \multicolumn{2}{|c|}{90 Days } & \multicolumn{2}{|c|}{45 Days } & \multicolumn{2}{|c|}{90 Days } \\
\hline & 2016 & 2017 & 2016 & 2017 & 2016 & 2017 & 2016 & 2017 \\
\hline \multicolumn{9}{|l|}{ Green manuring } \\
\hline Without GM & $5.13(32)$ & $4.70(29)$ & $7.35(72)$ & $5.27(51)$ & $2.86(9)$ & $1.28(9)$ & $1.76(4)$ & $1.80(3)$ \\
\hline $\begin{array}{l}\text { GM with } 50 \mathrm{~kg} C . \\
\text { juncea } \mathrm{ha}^{-1}\end{array}$ & $5.40(36)$ & $5.22(35)$ & 7.59 (79) & $6.23(76)$ & $2.17(5)$ & $1.00(0)$ & $1.38(1)$ & $1.50(2)$ \\
\hline $\begin{array}{l}\text { GM with } 100 \mathrm{~kg} C \text {. } \\
\text { juncea } \mathrm{ha}^{-1}\end{array}$ & $5.69(42)$ & $6.13(51)$ & $7.85(87)$ & $7.27(107)$ & $2.03(4)$ & $1.83(3)$ & $1.68(3)$ & $1.48(2)$ \\
\hline $\operatorname{LSD}(P=0.05)$ & 0.27 & 0.66 & 0.31 & 0.71 & 0.13 & 0.12 & 0.16 & 0.21 \\
\hline \multicolumn{9}{|l|}{ Weed control } \\
\hline Weedy & $8.84(78)$ & $9.86(98)$ & $14.2(201)$ & $15.97(262)$ & $3.80(14)$ & $1.84(4)$ & $2.92(9)$ & $2.16(4)$ \\
\hline Weed Free & $1.00(0)$ & $1.00(0)$ & $1.00(0)$ & $1.00(0)$ & $1.00(0)$ & $1.00(0)$ & $1.00(0)$ & $1.00(0)$ \\
\hline $\begin{array}{l}\text { Penoxsulam } 22.5 \mathrm{~g} \\
\mathrm{ha}^{-1}\end{array}$ & 5.35 (28) & $6.16(38)$ & $6.52(42)$ & 7.06 (49) & $2.13(4)$ & $1.64(2)$ & $1.00(0)$ & $1.96(3)$ \\
\hline $\begin{array}{l}\text { Penoxsulam } 22.5 \mathrm{~g} \\
\mathrm{ha}^{-1}+\mathrm{HW}\end{array}$ & $6.44(40)$ & $4.39(18)$ & $8.67(74)$ & $1.00(0)$ & $2.48(6)$ & $1.00(0)$ & $1.50(2)$ & $1.25(1)$ \\
\hline LSD (P=0.05) & 0.31 & 0.38 & 0.26 & 0.57 & 0.16 & 0.12 & 0.13 & 0.16 \\
\hline $\begin{array}{l}\text { Interaction } \quad \text { LSD } \\
(P=0.05)\end{array}$ & 0.53 & 0.65 & 0.46 & 0.99 & 0.28 & 0.21 & 0.22 & 0.30 \\
\hline
\end{tabular}

Parentheses are original values; daysa subjected to square root transformation before analysis

Table 8: Interaction effect of green manuring and weed control treatments on grass weed biomass at 45 days in 2016

\begin{tabular}{|c|c|c|c|c|}
\hline \multirow{2}{*}{$\begin{array}{l}\text { Green manuring/ } \\
\text { weed control }\end{array}$} & \multicolumn{4}{|c|}{ Grass weed biomass at 45 days $\left(\mathrm{g} \mathrm{m}^{-2}\right)$} \\
\hline & Weedy & Weed free & Penoxsulam & Penoxsulam+ HW \\
\hline Without GM & $7.82(60)$ & $1.00(0)$ & $5.60(30)$ & $6.10(36)$ \\
\hline GM with $50 \mathrm{~kg} \mathrm{C}$. juncea ha-1 & $8.66(74)$ & $1.00(0)$ & $5.30(27)$ & $6.66(43)$ \\
\hline GM with $100 \mathrm{~kg} C$. juncea ha-1 & $10.05(100)$ & $1.00(0)$ & $5.16(26)$ & $6.56(42)$ \\
\hline
\end{tabular}

The lower weed biomass under GM than without green manure may partly be attributed to allelopathy and smothering effects of green manure vegetation which suppressed weed growth as explained above under weed density heading and partly to the lower weed density under GM than without green manure treatments (Tables 7, 2 and 4).

The study concluded that sunnhemp green manuring promotes germination of aerobic weeds and hence could reduce weed pressure only in rice fields cultivated under water scarcity conditions.

Among green manure treatments, in 2016, GM-100 had a significantly higher seed bank of Echinochloa crus-galli at the time of incorporation (45 DAS; rice transplanting) while GM-50 had a significantly higher seed bank than without GM. The interaction effect revealed that at 45 DAYS, GM-100 under weedy check accumulated the highest grass weed biomass which was significantly higher than all other treatment combinations in both years (Tables 8 and 9).

Table 9: Interaction effect of green manuring and weed control treatments on grass weed biomass at 45 days in 2017

\begin{tabular}{|c|c|c|c|c|}
\hline \multirow{2}{*}{$\begin{array}{l}\text { Green manuring/ } \\
\text { weed control }\end{array}$} & \multicolumn{4}{|c|}{ Grass weed biomass at 45 days $\left(\mathrm{g} \mathrm{m}^{-2}\right)$} \\
\hline & Weedy & Weed free & Penoxsulam & Penoxsulam+ HW \\
\hline Without GM & $8.89(78)$ & $1.00(0)$ & $5.04(24)$ & $3.87(14)$ \\
\hline GM with $50 \mathrm{~kg}$ C. juncea ha $^{-1}$ & $9.40(88)$ & $1.00(0)$ & $5.65(31)$ & $4.83(22)$ \\
\hline GM with $100 \mathrm{~kg} C$. juncea ha $^{-1}$ & $11.27(127)$ & $1.00(0)$ & $7.78(60)$ & $4.48(19)$ \\
\hline \multicolumn{5}{|l|}{$\operatorname{LSD}(P=0.05)$} \\
\hline
\end{tabular}

Grain yield: The effects of GM and weed control treatments on rice grain yield were significant in both years. In 2016, penoxsulam with GM-100 combination gave the highest grain yield which was at par with penoxsulam with GM-50 as well as, 
without GM, weed-free with GM-50 as well as without GM. Penoxsulam alone gave significantly higher grain yield than penoxsulam plus HW at all levels of GM. Rice grain yield under weed-free without GM combination was significantly higher than penoxsulam plus HW at all levels of GM. Under the weedy check, the grain yields were similar at all levels of GM, however, the grain yields were significantly higher than all other treatment combinations except weedy check without GM and penoxsulam plus HW with GM-50 as well as GM-100 combinations which were at par. In 2017, penoxsulam plus HW and without GM combination gave the highest grain yield which was significantly higher than all other treatment combinations (Table 10).

Table 10: Effect of green manuring and weed control treatments on rice grain yield in 2016

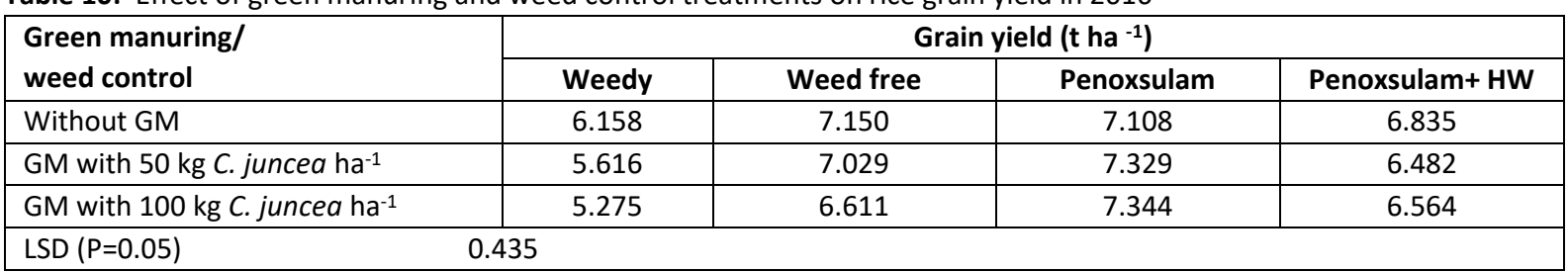

Penoxsulam plus HW gave significantly higher grain yield than penoxsulam alone and weed free, at all levels of GM. Under weedy check, the crop raised without GM gave significantly higher than GM-100 but it was at par with GM-50; GM-50 gave significantly higher grain yield than GM-100. Under weed-free, the grain yields under all levels of GM were at par while in case of penoxsulam alone the grain yield without GM was significantly higher than with GM-50 and GM-100 (Table 11).

Table 11: Effect of green manuring and weed control treatments on rice grain yield in 2017

\begin{tabular}{|l|c|c|c|c|}
\hline \multirow{2}{*}{$\begin{array}{l}\text { Green manuring/ } \\
\text { weed control }\end{array}$} & Weedy & Weed free & Prain yield $\left(\mathbf{t ~ h a ~}^{-1}\right)$ \\
\cline { 2 - 5 } Without GM & 4.987 & 6.553 & 7.194 & Penoxsulam+ HW \\
\hline GM with 50 kg C. juncea ha $^{-1}$ & 4.552 & 6.812 & 6.636 & 7.245 \\
\hline GM with 100 kg C. juncea ha ${ }^{-1}$ & 3.365 & 6.465 & 6.255 & 7.574 \\
\hline LSD (P=0.05) & 0.437 \\
\hline
\end{tabular}

The result of the study indicated that in puddle transplanted rice, raised with recommended doses of fertilizers, the green manuring with $C$. juncea did not provide any additional benefits in terms of weed control and or rice grain yield as compared to the crop raised without green manure, and that penoxsulam alone or followed by one hand weeding could be adopted for effective control of weeds in this crop.

\section{DISCUSSION}

The allelopathic effect of $C$. juncea on weeds has been reported earlier (Skinner, 2012). Kumar and Ladha (2011), reported lower weed emergence under GM as compared to without green manure. Chauhan and Johnson (2008), indicated that GM was more effective on weed species present in topsoil layers, light-responsive and having low dormancy. In this context, the present study has support from Chauhan and Johnson (2008), concerning density of $D$. ciliaris and $E$. indica, however, the results are in contrast for $C$. rotundus whose tubers are placed deep in the soil thus indicating that sunnhemp green manuring may help reduce the density of perennial weeds also. Further, the results indicated that sunnhemp green manuring could be more helpful in reducing the density of aerobic weed species.

Earlier studies by Buhler (2002), and Moonen and Barberi (2004), had reported lower weed establishment and weed biomass under cover crop as compared to without cover crop. Cherr et al., (2006), reported that C. juncea had high C:N and produce high residue levels which results in higher weed suppression for a longer period than cover crops with low $\mathrm{C}$ : $\mathrm{N}$ ratios. Putnam and De Frank (1983), and Mangan et al (1995), had reported that green manure crops, owing to their fast growth rate, change the micro environment through release of phytotoxins which influences weed germination and establishment. Teasdale and Daughtry (1993) found the cover cropping of Vicia villosa reduced weed density by $74 \%$ and weed biomass by $61 \%$ of as compared to without cover crop. The cover crop residue near to $4.5 \mathrm{t} \mathrm{ha}^{-1}$ of biomass results in weed suppression (Balkcom et al., 2007). In the current study, the GM produced 4.3 to $4.8 \mathrm{t} \mathrm{ha}^{-1}$ at both seed rates which suppressed weed growth and weed biomass. According to Cherr et al., (2006), C. juncea exhibit its suppressive effect on weeds by reducing the light penetration at the soil surface while Adler and Chase (2007), attributed that the suppression is through chemical extracts. The beneficial effect of green manure crops in reducing soil weed seed bank has been reported earlier (Kumar and Ladha 2011; Moonen and Barberi 2004).

\section{CONCLUSIONS}

The effects of Crotalaria juncea green manure and penoxsulam herbicide on weeds and productivity of transplanted rice were investigated in a field study conducted over two cropping seasons, 2016 and 2017, at Ludhiana. The green manure was incorporated at 45 days after sowing green manuring with 50 and $100 \mathrm{~kg} C$. juncea seed ha-1 had significantly lower density and biomass of Cyperus rotundus, Digitaria ciliaris and Eleusine indica as compared to without green manure in both years. In transplanted rice fields, the crop raised after green manuring with 50 and $100 \mathrm{~kg} C$. juncea seed ha-1 treatments had higher 
density and biomass of Echinochloa crus-galli and Echinochloa colona than without green manure. Green manuring favoured the build-up of seed bank of E. crus-galli while E. colona seed bank results were not consistent. Penoxsulam $22.5 \mathrm{~g}$ ha-1 as early post-emergence (12 DAYS), alone and followed by hand weeding (45 DAYS), gave significantly better weed control than weedy check in both years; penoxsulam alone had significantly better weed control than when it was followed by one hand weeding in 2016 while the reverse was true in 2017. In 2016, penoxsulam $22.5 \mathrm{~g} \mathrm{ha}^{-1}$ and $100 \mathrm{~kg} \mathrm{C}$. juncea seed ha-1 treatment combination gave the highest rice grain yield which was at par to penoxsulam $22.5 \mathrm{~g} \mathrm{ha}^{-1}$ with either of without green manure and $50 \mathrm{~kg} \mathrm{C}$. juncea seed ha-1, weed free with either of without green manure and $50 \mathrm{~kg}$ C. juncea seed ha-1. In 2017, penoxsulam $22.5 \mathrm{~g} \mathrm{ha}^{-1}$ plus hand weeding and without green manuring combination gave the highest rice grain yield which was significantly higher than all other treatment combinations. A similar trend was followed in the case of rice straw yield.

In summary, in puddle transplanted rice raised with recommended doses of fertilizers, the green manuring with $C$. juncea did not provide any additional benefits in terms of weed control and or rice grain yield as compared to the crop raised without green manure, and penoxsulam $22.5 \mathrm{~g} \mathrm{ha}^{-1}$ alone or followed by one hand weeding gave effective weed control.

\section{REFERENCES}

Adler, M. J., \& Chase, C. A. (2007). Comparison of the allelopathic potential of leguminous summer cover crops: cowpea, sunn hemp, and velvetbean. HortScience, 42(2), 289-293.

Balkcom, K., Schomberg, H., Reeves, W., Clark, A., Baumhardt, L., Collins, H., \& Mitchell, J. (2007). Managing cover crops in conservation tillage systems. Managing cover crops profitably, 44-61.

Buhler, D. D. (2002). 50th Anniversary-Invited Article: Challenges and opportunities for integrated weed management. Weed Science, 50(3), 273-280.

Chauhan, B. S., \& Johnson, D. E. (2008). Germination ecology of Chinese sprangletop (Leptochloa chinensis) in the Philippines. Weed Science, 56(6), 820-825.

Chauhan, B. S., \& Johnson, D. E. (2011). Growth response of direct-seeded rice to oxadiazon and bispyribac-sodium in aerobic and saturated soils. Weed Science, 59(1), 119-122.

Cherr, C. M., Scholberg, J. M. S., \& McSorley, R. (2006). Green manure as nitrogen source for sweet corn in a warm-temperate environment. Agronomy Journal, 98(5), 1173-1180.

Deepanshi, R., Monika, M. B., \& Nidhi, V. (2020). Role of microsatellite markers in molecular diversity analysis of rice bean (Vigna umbellta). Asian Journal of Bio Science, 15(1), 15-20.

Ghosh, P. K., Bandyopadhyay, K. K., Wanjari, R. H., Manna, M. C., Misra, A. K., Mohanty, M., \& Rao, A. S. (2007). Legume effect for enhancing productivity and nutrient use-efficiency in major cropping systems-an Indian perspective: a review. Journal of Sustainable Agriculture, 30(1), 59-86.

He, H. B., Li, W. X., Zhang, Y. W., Cheng, J. K., Jia, X. Y., Li, S., \& Xin, G. R. (2020). Effects of Italian ryegrass residues as green manure on soil properties and bacterial communities under an Italian ryegrass (Lolium multiflorum L.)-rice (Oryza sativa L.) rotation. Soil and Tillage Research, 196, 104487.

Kumar, A., Singh, R. K. P., Singh, K. M., \& Mishra, J. S. (2018). Economics of paddy (Oryza sativa) production: A comparative study of Bihar and Punjab. Indian Journal of Agricultural Sciences, 88(2), 314-319.

Kumar, V., \& Ladha, J. K. (2011). Direct seeding of rice: recent developments and future research needs. Advances in Agronomy, 111, 297-413.

Longa, C. M., Nicola, L., Antonielli, L., Mescalchin, E., Zanzotti, R., Turco, E., \& Pertot, I. (2017). Soil microbiota respond to green manure in organic vineyards. Journal of Applied Microbiology, 123(6), 1547-1560.

Moonen, A. C., \& Barberi, P. (2004). Size and composition of the weed seedbank after 7 years of different cover-crop-maize management systems. Weed Research, 44(3), 163-177.

Marshall, C. B., \& Lynch, D. H. (2020). Soil microbial and macrofauna dynamics under different green manure termination methods. Applied Soil Ecology, 148, 103505.

Manhas, S. S., Singh, G., Singh, D., \& Khajuria, V. (2012). Effect of tank-mixed herbicides on weeds and transplanted rice (Oryza sativa L.). Annals of Agricultural Research, 33(12), 12-20.

Mangan, F., DeGregorio, R., Schonbeck, M., Herbert, S., Guillard, K., Hazzard, R., \& Litchfield, G. (1995). Cover cropping systems for brassicas in the Northeastern United States: 2. Weed, insect and slug incidence. Journal of Sustainable Agriculture, 5(3), 15-36.

Maguire, V. G., Bordenave, C. D., Nieva, A. S., Llames, M. E., Colavolpe, M. B., Gárriz, A., \& Ruiz, O. A. (2020). Soil bacterial and fungal community structure of a rice monoculture and rice-pasture rotation systems. Applied Soil Ecology, 151, 103-535.

Pratt, O. J., \& Wingenbach, G. (2016). Factors affecting adoption of green manure and cover crop technologies among Paraguayan smallholder farmers. Agroecology and Sustainable Food Systems, 40(10), 1043-1057.

Putnam, A. R., \& DeFrank, J. (1983). Use of phytotoxic plant residues for selective weed control. Crop protection, 2(2), 173181.

Qaswar, M., Huang, J., Ahmed, W., Li, D., Liu, S., Ali, S., \& Zhang, H. (2019). Long-term green manure rotations improve soil biochemical properties, yield sustainability and nutrient balances in acidic paddy soil under a rice-based cropping system. Agronomy, 9(12), 780.

Raviv, M. (2015, October). Can the use of composts and other organic amendments in horticulture help to mitigate climate change?. In II International Symposium on Organic Matter Management and Compost Use in Horticulture 1076 (pp. 1928). 
Recalde, K. M. G., Carneiro, L. F., Carneiro, D. N. M., Felisberto, G., Nascimento, J. S., \& Padovan, M. P. (2015). Weed suppression by green manure in an agroecological system1. Revista Ceres, 62, 546-552.

Raheem, A., Zhang, J., Huang, J., Jiang, Y., Siddik, M. A., Deng, A., ... \& Zhang, W. (2019). Greenhouse gas emissions from a rice-rice-green manure cropping system in South China. Geoderma, 353, 331-339.

Sheng-nan, C. H. E. N., Jun-ming, H. U., Xian-li, X. U., Xiang-hua, W. E. I., \& Tie-guang, H. E. (2018). Effect of Smash Ridging Conservation Tillage with Green Manure on Rice Field Soil Infiltration and Its Delayed Action. Chinese Journal of Agrometeorology, 39(12), 778.

Songjuan, G. A. O., Weidong, C. A. O., \& Guopeng, Z. H. O. U. (2021). Bacterial communities in paddy soils changed by milk vetch as green manure: A study conducted across six provinces in South China. Pedosphere, 31(4), 521-530.

Singh, P., Singh, P., Singh, R., \& Singh, K. N. (2007). Efficacy of new herbicides in transplanted rice (Oryza sativa) under temperate conditions of Kashmir. Indian Journal of Weed Science, 39(3and4), 167-171.

Skinner, E. M., Díaz-Pérez, J. C., Phatak, S. C., Schomberg, H. H., \& Vencill, W. (2012). Allelopathic effects of sunnhemp (Crotalaria juncea L.) on germination of vegetables and weeds. HortScience, 47(1), 138-142.

Teasdale, J. R., \& Daughtry, C. S. (1993). Weed suppression by live and desiccated hairy vetch (Vicia villosa). Weed Science, 41(2), 207-212.

Thiessen Martens, J. R., Lynch, D. H., \& Entz, M. H. (2019). A survey of green manure productivity on dryland organic grain farms in the eastern prairie region of Canada. Canadian Journal of Plant Science, 99(5), 772-776.

Zhou, G., Gao, S., Lu, Y., Liao, Y., Nie, J., \& Cao, W. (2020). Co-incorporation of green manure and rice straw improves rice production, soil chemical, biochemical and microbiological properties in a typical paddy field in southern China. Soil and Tillage Research, 197, 104499. 


\title{
تأثير السماد الأخضر (Crotalaria juncea L. القائمة على الأرز المزروع بالشتل
}

\author{
1 ذبيح الله جلالي 2 * ومحمد حسين فلح زادة ،

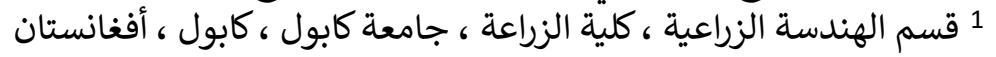

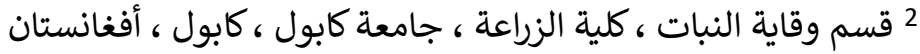

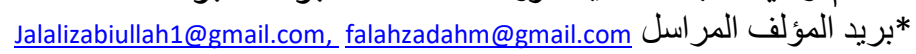

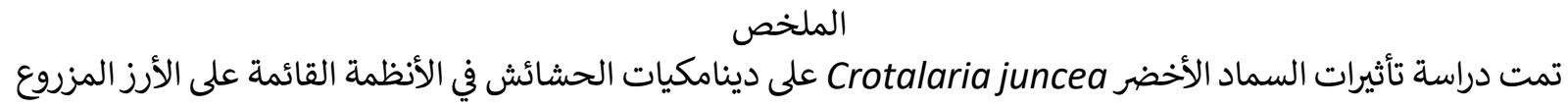

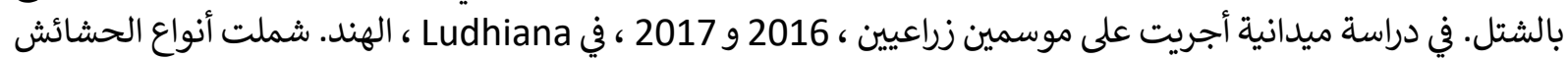
الرئيسية في الحقل ، خلال مرحلة التسميد الأخضر ، Digitaria ciliaris و Cyperus rotundus و و

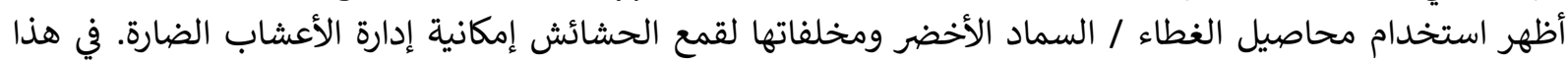

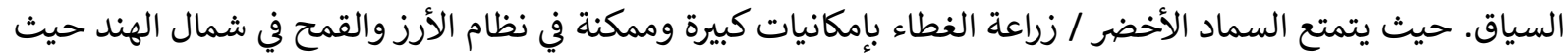

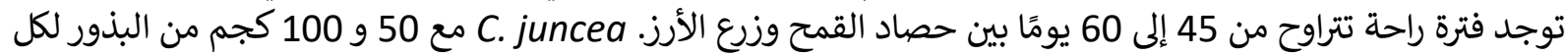

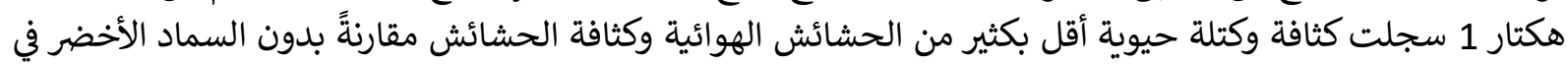

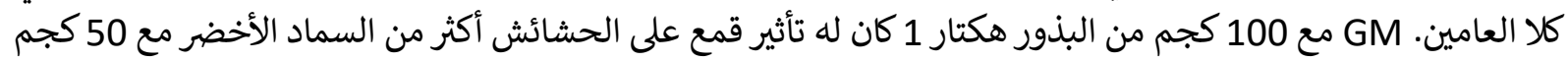

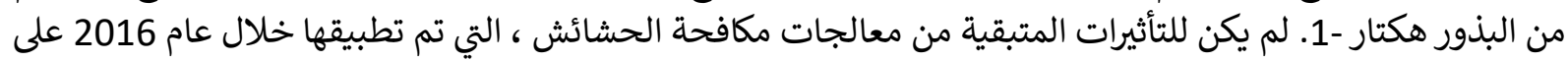

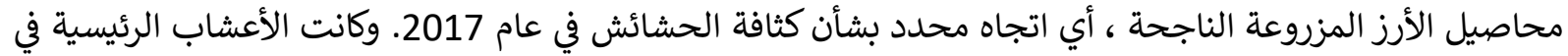

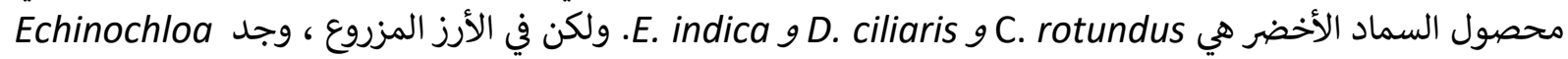
E و colona الكلمات المفتاحية: السماد الأخضر ، دينامكيات الحشائش ، الحشائش الهوائية ، الأرز 